\title{
LOCALLY CONVEX LINEAR TOPOLOGICAL SPACES THAT ARE HOMEOMORPHIC TO THE POWERS OF THE REAL LINE
}

\author{
A. CHIGOGIDZE
}

(Communicated by James E. West)

\begin{abstract}
We give a characterization of locally convex linear topological spaces that are homeomorphic to the uncountable powers of the real line.
\end{abstract}

\section{INTRODUCTION}

One of the fundamental results of infinite-dimensional topology and functional analysis-the well-known theorem of Anderson-Kadets (see, e.g. [2]) states that each separable infinite-dimensional Frechet space is homeomorphic to the countable (infinite) power $R^{\omega}$ of the real line $R$. It is also well known [2] that each locally compact locally convex linear topological space is homeomorphic (even isomorphic) to some finite power $R^{n}$ of $R$ (in this paper we consider only Hausdorff linear topological spaces over the field of real numbers). The following reformulation of these two facts immediately follows from the corresponding classical definitions (let us recall only that the Polish spaces are defined as separable and completely metrizable ones; of course, each metrizable and locally compact space is Polish).

Theorem A. The following conditions are equivalent for each locally convex linear topological space $E$ :

(i) $E$ is homeomorphic to the countable (finite or infinite) power of the real line;

(ii) $E$ is a Polish space.

The main purpose of this paper is to characterize those locally convex linear topological spaces that are homeomorphic to uncountable powers of the real line. Moreover, we want to find simple (purely topological) conditions that will give the desired homeomorphisms. The main idea we use for getting such a characterization states that we can consider our problem from the point of view of the general theory of absolute extensors in the dimension 0 . Let us outline in some words the last notion. Without formalities the definition of this notion is

Received by the editors February 23, 1990.

1980 Mathematics Subject Classification (1985 Revision). Primary 57N17, 54C55. 
as follows: a topological space $X$ is said to be an absolute extensor in the dimension zero (shortly, $A E(0)$-space) if for any zero-dimensional space $Z$ and any subspace $Z_{0}$ of it each mapping $f: Z_{0} \rightarrow X$ can be extended to the whole of $Z$. Of course this is only a scheme of the correct definition. As a rule some additional restrictions in this definition are assumed (otherwise we can not obtain a satisfactory notion). Some of them restrict the class of spaces under consideration and the others specify the types of embeddings of mentioned subspaces into ambient spaces. Historically the basic classes of metrizable spaces and of compact spaces as well as closed embeddings were mainly considered. Many results in the case of metrizable spaces were obtained. We recall only one of them, Michael's selection theorem [2], which states that each completely metrizable space is an $A E(0)$-space in the class of all metrizable spaces. On the other hand, the first result concerned with the class of compact $A E(0)$-spaces and that was the starting point of the whole theory of nonmetrizable absolute extensors was proved only in 1974 . We have in mind the fundamental theorem of Haydon [9] stating that the class of compact $A E(0)$-spaces coincides with the class of so-called Dugundji compact spaces. After this result compact $A E(0)$-spaces were extensively studied and today we have a sufficiently developed theory of such spaces. Good examples of results of this theory are Shchepin's theorems concerning topological characterizations of uncountable powers of two-point discrete space and of the closed unit interval [10]. At the same time it should be observed that the standard definition of the concept of an $A E(0)$-space is satisfactory only for various subclasses of the class of normal spaces (see [3] for the complete discussion of corresponding reasons). This made it impossible to use this notion in studying nonmetrizable linear topological spaces and the associated questions of infinite-dimensional topology and functional analysis. The satisfactory modification of the notion of an $A E(0)$-space for the class of all Tychonov (i.e., completely regular and Hausdorff) spaces was given in [3]. Moreover, in [3] topological characterizations of uncountable powers of the real line and of the space of natural numbers were also obtained (let us recall that countable infinite powers of the above spaces were characterized correspondingly by Toruńczyk [11] and by Alexandrov and Urysohn [1]). Let us mention only one proposition [3] concerning arbitrary $A E(0)$-spaces (all exact definitions will be given below) that, together with Theorem A, can be considered as a key to a solution of our problem: the class of Polish spaces coincides with the class of all $A E(0)$-spaces with countable weight. Now we can formulate the main result of this paper which, together with Theorem A, gives a complete topological characterization of those locally convex linear spaces which are homeomorphic to powers of the line.

Theorem B. Let $\tau$ be an uncountable cardinal. Then the following conditions are equivalent for each locally convex linear topological space $E$ :

(i) $E$ is homeomorphic to $R^{\tau}$;

(ii) $E$ is an $A E(0)$-space of weight $\tau$. 
We also prove some other propositions and corollaries of Theorem B.

Preliminaries. All spaces below are assumed to be Tychonov and all mappings continuous. $C(X)$ denotes the set of all real-valued continuous functions defined on the topological space $X$. If $X \subseteq Y$ then $C(Y) \mid X$ is the set of all elements of $C(X)$ extendable to the whole of $Y$. The equality $C(X)=C(Y) \mid X$ characterizes the $C$-embedded subspaces. If $f: X \rightarrow Y$ is a mapping then $C(f): C(Y) \rightarrow C(X)$ denotes the naturally obtained operator. By dimension we mean the dimension dim, which is defined by means of finite functionally open coverings [7]. (A set is functionally open provided that it is the pre-image of an open subset of the reals of a map $f \in C(X)$.) In the class of compact spaces the following notions were defined by Shchepin [10]. In the present form they were given in [3].

Defintion 1. A mapping $f: X \rightarrow Y$ is said to be $n$-soft $(n=0,1, \ldots, \infty)$ if, for any space $Z$ of dimension $\operatorname{dim} Z \leq n$, any two subspaces $Z_{0}$ and $Z_{1}$ of it such that $Z_{0} \subseteq Z_{1}$, and any mappings $g: Z_{0} \rightarrow X$ and $h: Z_{1} \rightarrow Y$ such that $C(g)(C(X)) \subseteq C(Z)\left|Z_{0}, C(h)(C(Y)) \subseteq C(Z)\right| Z_{1}$, and $f g=h \mid Z_{0}$, there exists a mapping $k: Z_{1} \rightarrow X$ such that $f k=h$, and $C(k)(C(X)) \subseteq C(Z) \mid Z_{1}$. The $\infty$-soft mappings will be called soft mappings.

Definition 2. A space $X$ is called an absolute extensor in dimension $n(n=$ $0,1, \ldots, \infty)$, briefly an $A E(n)$-space, if its constant map is $n$-soft. The $A E(\infty)$ spaces are called absolute extensors.

It follows from [3] that in the class of compact spaces as well as in the class of Polish spaces the above definitions agree with the common definitions of the corresponding concepts. $A E(n)$-spaces as well as $n$-soft mappings between them can be characterized in various ways. Very useful for their investigation are spectral methods. Let us recall some necessary definitions. Suppose that $A$ is a partially ordered directed set (this means that for every two elements $\alpha, \beta \in$ $A$ there exists an element $\gamma \in A$ such that $\gamma \geq \alpha$ and $\gamma \geq \beta$ ). A subset of $A$ is said to be a chain if every two elements of it are comparable. The symbol sup $B$, where $B \subseteq A$, denotes the lower upper bound of $B$ ( of course in the case when such element exists in $A$ ). A subset $B$ of $A$ is said to be closed in $A$ if for each chain $C$ in $B$ we have sup $C \in B$ whenever an element sup $C$ exists in $A$. Finally a directed set $A$ is said to be $\lambda$-complete (where $\lambda$ is an infinite cardinal) if for every chain $B$ of elements of $A$ with $|B| \leq \lambda$ there exists an element sup $B$ in $A$. We obtain the standard example of a $\lambda$-complete set if consider the set $\exp _{\lambda} A$ of all subsets of cardinality $\leq \lambda$ of an arbitrary set $A$ with $|A|>\lambda$. Now suppose that we have an inverse spectrum $S_{X}=\left\{X_{\alpha}, p_{\alpha}^{\beta}, A\right\}$. By $\lim S_{X}$ and by $p_{\alpha}, \alpha \in A$, we denote the limit space of our spectrum and the natural limit projections from $\lim S_{X}$ to $X_{\alpha}$ respectively. If we have a cofinal subset $A^{\prime}$ of $A$ then the limit space of the spectrum $S_{X} \mid A^{\prime}=\left\{X_{\alpha}, p_{\alpha}^{\beta}, \alpha, \beta, \in A^{\prime}\right\}$ is naturally homeomorphic to $\lim S_{X}$. The spectrum $S_{X}$ is said to be continuous if for each chain $B$ in $A$ with $\sup B=\beta$ the diagonal product of mappings 
$p_{\alpha}^{\beta}, \alpha \in B$, maps the space $X_{\beta}$ onto $\lim S_{X} \mid B$ homeomorphically. If we have two inverse specta $S_{X}=\left\{X_{\alpha}, p_{\alpha}^{\beta}, A\right\}$ and $S_{Y}=\left\{Y_{\alpha}, q_{\alpha}^{\beta}, A\right\}$ over the same indexed sets and a morphism between them, i.e., the collection $\left\{f_{\alpha}, \alpha \in A\right\}$ of mappings $f_{\alpha}: X_{\alpha} \rightarrow Y_{\alpha}, \alpha \in A$, such that for each pair of indexes $\alpha, \beta \in A$ the equality $q_{\alpha}^{\beta} f_{\beta}=f_{\alpha} p_{\alpha}^{\beta}$ holds whenever $\alpha \leq \beta$, then there exists a limit mapping $\lim \left\{f_{\alpha}: \alpha \in A\right\}: \lim S_{X} \rightarrow \lim S_{Y}$ of this morphism which is defined as a diagonal product of mappings $f_{\alpha} p_{\alpha}: \lim S_{X} \rightarrow Y_{\alpha}, \alpha \in A$, and satisfies the equalities $q_{\alpha}\left\{f_{\alpha}: \alpha \in A\right\}=f_{\alpha} p_{\alpha}$ for each $\alpha \in A$. The spectrum $S_{X}$ is said to be factorizing if for each function $f: \lim S_{X} \rightarrow R$ there exist an index $\alpha \in A$ and a function $f_{\alpha}: X_{\alpha} \rightarrow R$ such that $f=f_{\alpha} p_{\alpha}$. Finally a continuous spectrum $S_{X}$ is said to be a $\lambda$-spectrum if its index set is $\lambda$-complete and if the spaces $X_{\alpha}, \alpha \in A$, have weight $\leq \lambda$.

The following result for compact spaces was proved by Haydon [9] (the case $n=0$ ), Shchepin [10] (the cases $n=0, \infty$ ), Fedorchuk [8] (the case $n=1$ ), and Dranishnikov [6] (the cases $n \geq 2$ ). The following theorem that was proved in [3] generalizes all mentioned results and gives even the characterization of $n$-soft mappings between arbitrary $A E(n)$-spaces.

Theorem 1. A mapping $f: X \rightarrow Y$ between $A E(n)$-spaces $(n=0,1, \ldots, \infty)$ is $n$-soft if and only if there exists factorizing $\omega$-spectra $S_{X}$ and $S_{Y}$ consisting of Polish spaces and a morphism $\left\{f_{\alpha}\right\}: S_{X} \rightarrow S_{Y}$ consisting of n-soft mappings such that the following conditions are satisfied:

(i) $X=\lim S_{X}, Y=\lim S_{Y}$, and $f=\lim f_{\alpha}$;

(ii) all the limit projections in the spectra $S_{X}$ and $S_{Y}$ are $n$-soft:

(iii) all the limit square diagrams are $n$-soft;

(iv) if $f$ itself has a Polish kernel, then all the limit square diagrams are Cartesian squares.

Taking the $Y$ in this theorem to be a singleton space, we get a spectral characterization of $A E(n)$-spaces.

Now we shall comment some special notions concerning the above theorem. By the limit square diagram associated with mapping $f$ and the corresponding morphism we understand the diagram of the following type

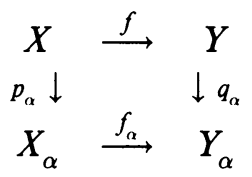

where $\alpha \in A$. Each such commutative square diagram naturally induces the following diagram of more complicated type:

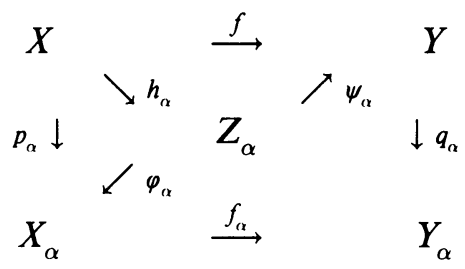


Here $Z_{\alpha}$ denotes the fibered product of $X_{\alpha}$ and $Y$ with respect to mappings $f_{\alpha}$ and $q_{\alpha}$ (in the other words $Z_{\alpha}$ is a subspace of the product consisting exactly of those points $\left(x_{\alpha}, y\right) \in X_{\alpha} \times Y$ for which the equality $f_{\alpha}\left(x_{\alpha}\right)=q_{\alpha}(y)$ holds); $\varphi_{\alpha}: Z_{\alpha} \rightarrow X_{\alpha}$ and $\psi_{\alpha}: Z_{\alpha} \rightarrow Y$ denote the restrictions to $Z_{\alpha}$ of the natural projections of $X_{\alpha} \times Y$ onto the first and second factors respectively; the mapping $h_{\alpha}: X \rightarrow Z_{\alpha}$ denotes the characteristic mapping of the first diagram and is defined to be the diagonal product of $p_{\alpha}$ and $f$. It is clear that the characteristic mapping is a homeomorphism precisely for diagrams that are Cartesian squares (i.e., pullback squares). But in general they are not even surjective. A commutative square diagram is said to be $n$-soft if its characteristic mapping is $n$-soft. The following notion of mapping with Polish kernel was defined in [3]. It generalizes the notion of mapping with metrizable kernel introduced in [9]. A mapping $f: X \rightarrow Y$ has a Polish kernel if there exists a Polish space $P$ such that $X$ is $C$-embedded in $Y \times P$ and $f$ coincides with the restriction of the projection $\pi_{Y}$ to $X$.

Theorem 1 indicates a general method of studying nonmetrizable $A E(n)$ spaces. If we want to prove some result for such spaces then our strategy can be as follows: first of all we must prove the corresponding result for Polish spaces; on the second step we must prove a parametrical version of the first one; after that we can use Theorem 1 and, constructing from our $\omega$-spectrum a well-ordered one, step by step (as a rule by transfinite induction) obtain the desired result.

\section{THE MAIN RESULT}

Now we can proceed to direct proofs of our results. We start with the following proposition.

Lemma 1. Let $\lambda \geq \omega$. Each locally convex $A E(0)$-space is isomorphic to the limit space of some factorizing $\lambda$-spectrum consisting of locally convex $A E(0)$ spaces (of weight $\leq \lambda$ ) and 0-soft linear limit projections. In particular, each locally convex $A E(0)$-space is isomorphic to the limit space of some factorizing $\omega$-spectrum consisting of separable Fréchet spaces and 0 -soft linear limit projections.

Proof. Let $E$ be a locally convex $A E(0)$-space. It follows directly from Theorem 1 that we can represent (topologically!) the space $E$ as the limit space of some factorizing $\lambda$-spectrum $S=\left\{E_{\alpha}, p_{\alpha}^{\beta}, A\right\}$ consisting of $A E(0)$-spaces and 0 -soft limit projections. Let us show that this spectrum contains a closed and cofinal subspectrum (this means that there exists a closed and cofinal subset $A^{\prime}$ that produces the desired subspectrum) consisting precisely of locally convex spaces and 0-soft linear limit projections. Obviously this will be sufficient for us.

Consider the product $E \times E$ and the spectrum $S \times S=\left\{E_{\alpha}^{2}, p_{\alpha}^{\beta} \times p_{\alpha}^{\beta}, A\right\}$. Clearly $\lim (S \times S)=E \times E$. It follows from [3] that each 0-soft mapping 
between $A E(0)$-spaces is open. Consequently all limit projections $p_{\alpha} \times p_{\alpha}, \alpha \in$ $A$, of the spectrum $S \times S$ are open. By [10] we can conclude that the spectrum $S \times S$ is also factorizing. Let us additionally note that each 0 -soft mapping is surjective [3]. Denote by $\mu: E \times E \rightarrow E$ a continuous law of addition that is given in $E$ as in the linear topological space. In our situation we can use the spectral theorem of Shchepin [10] to obtain a closed and cofinal subset $A_{1}$ of $A$ and a morphism $\left\{\mu_{\alpha}: E_{\alpha} \times E_{\alpha} \rightarrow E_{\alpha}, A_{1}\right\}:(S \times S)\left|A_{1} \rightarrow S\right| A_{1}$ whose limit mapping coincides with the mapping $\mu$. Now we can define a continuous law $+_{\alpha}$ of addition in each $E_{\alpha}, \alpha \in A_{1}$. For this we put $x_{\alpha}+{ }_{\alpha} y_{\alpha}=\mu_{\alpha}\left(x_{\alpha}, y_{\alpha}\right)$ whenever $\left(x_{\alpha}, y_{\alpha}\right) \in E_{\alpha} \times E_{\alpha}$.

Similarly we can consider a factorizing $\lambda$-spectrum

$$
\operatorname{id} \times S=\left\{R \times E_{\alpha}, \operatorname{id}_{R} \times p_{\alpha}^{\beta}, A\right\}
$$

and continuous law of multiplication of elements of $E$ on scalars (i.e., real numbers). Denote this law by $\nu: R \times E \rightarrow E$. Then as above we get a closed and cofinal subspectrum $S \mid A_{2}$ of $S$ and a morphism $\left\{\nu_{\alpha}: R \times E_{\alpha} \rightarrow\right.$ $\left.E_{\alpha}, A_{2}\right\}:(\operatorname{id} \times S)\left|A_{2} \rightarrow S\right| A_{2}$ with $\nu=\lim \left\{\nu_{\alpha}, \alpha \in A_{2}\right\}$. We can look on the mapping $\nu_{\alpha}$ as on the continuous law of multiplication on scalars in $E_{\alpha}$. Let us note now that the set $A^{\prime}=A_{1} \cap A_{2}$ is also a closed and cofinal subset of $A$ (we use the $\lambda$-completeness of $A$; see [10]). Hence the subspectrum $S \mid A^{\prime}$ of $S$ is also a factorizing $\lambda$-spectrum. But for spaces $E_{\alpha}$ with $\alpha \in A^{\prime}$ we have both operations from the definition of linear topological spaces. Both of them are continuous and consequently all spaces of the spectrum $S \mid A^{\prime}$ are linear topological spaces. Moreover all limit projections in the last spectrum are linear with respect to defined operations. The lemma is proved.

Corollary 1. Each locally convex $A E(0)$-space is isomorphic to a C-embedded linear subspace of the product of separable Fréchet spaces.

Proof. Let $E$ be a locally convex $A E(0)$-space. By Lemma $1, E$ is isomorphic to the limit space of some factorizing $\omega$-spectrum $S=\left\{E_{\alpha}, p_{\alpha}^{\beta}, A\right\}$ consisting of separable Fréchet spaces and 0 -soft linear projections. Consider the product $F=\prod\left\{E_{\alpha}: \alpha \in A\right\}$. Evidently $E$ is isomorphic to a linear (and closed) subspace of $F$ (this subspace is an image of the space $E$ under the diagonal product of all limit projections of the spectrum $S$ ). Since $S$ is factorizing the mentioned subspace is $C$-embedded in $F$. The proof is complete.

Lemma 2. Let $f: E \rightarrow F$ be a 0-soft linear mapping between locally convex $A E(0)$-spaces that has a Polish kernel (in the above-mentioned sense). Suppose that $f(K)=T$ where $K$ and $T$ are convex and $C$-embedded $A E(0)$-subspaces of $E$ and $F$ respectively. Then the following conditions are equivalent:

(i) $f \mid K: K \rightarrow T$ is a 0 -soft mapping:

(ii) $f \mid K: K \rightarrow T$ is a soft mapping.

Proof. The nontrivial part is the implication (i) $\Rightarrow$ (ii). By Lemma 1 there exist factorizing $\omega$-spectra $S_{E}=\left\{E_{\alpha}, p_{\alpha}^{\beta}, A\right\}$ and $S_{F}=\left\{F_{\alpha}, q_{\alpha}^{\beta}, A\right\}$ consisting of separable Fréchet spaces and 0 -soft linear projections, the limit spaces of 
which are isomorphic to the spaces $E$ and $F$ respectively. By Theorem 1 we can suppose without loss of generality that $f$ is the limit mapping of some morphism $\left\{f_{\alpha}: E_{\alpha} \rightarrow F_{\alpha}, A\right\}: S_{E} \rightarrow S_{E}$ consisting of 0-soft mappings and such that all the corresponding limit square diagrams are Cartesian squares. Since $K$ is a $C$-embedded and closed subspace of $E$ we can conclude that the spectrum $S_{K}=\left\{K_{\alpha}=\operatorname{cl}_{E_{\alpha}}\left(p_{\alpha}(K)\right), p_{\alpha}^{\beta} \mid K_{\beta}, A\right\}$, the limit space of which coincides with $K$, is also a factorizing $\omega$-spectrum. Since $K$ is an $A E(0)$-space we can again use Theorem 1 (together with the spectral theorem from [10]) and suppose without loss of generality that for each $\alpha \in A$ the mapping $p_{\alpha} \mid K$ is 0 -soft and $p_{\alpha}(K)=K_{\alpha}$. Similarly we can suppose that $q_{\alpha}(T)=\operatorname{cl}_{F_{\alpha}}\left(q_{\alpha}(T)\right)$ and the mapping $q_{\alpha} \mid T$ is 0 -soft whenever $\alpha \in A$. Finally by Theorem 1 we can suppose (again without loss of generality) that each commutative square diagram of the following type $(\alpha \in A)$

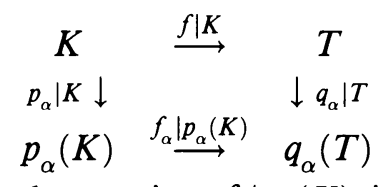

is a Cartesian square and each mapping $f_{\alpha} \mid p_{\alpha}(K)$ is 0 -soft. In this situation to conclude the proof of the lemma it remains to prove that the last mapping is soft (then we can use the fact that the above diagram is a Cartesian square to obtain the softness of the mapping $f \mid K)$.

Linearity of the limit projections of spectra $S_{E}$ and $S_{F}$ imply that the spaces $p_{\alpha}(T)$ and $q_{\alpha}(T)$ are convex subspaces of the spaces $E_{\alpha}$ and $F_{\alpha}$ respectively. Moreover it is easy to see that each mapping $f_{\alpha}$ is also linear. By Michael's selection theorem [2] the mapping $f_{\alpha} \mid p_{\alpha}(K)$, as an open mapping with closed convex fibers between closed and convex subspaces of separable Fréchet spaces, is soft. The proof is complete.

Lemma 3. Let $K$ be a $C$-embedded and convex subspace of a loally convex $A E(0)$-space. Then the following conditions are equivaient:

(i) $K$ is an $A E(0)$-space;

(ii) $K$ is an absolute extensor.

Proof. It is sufficient to prove an implication (i) $\Rightarrow$ (ii). By Corollary 1 we can suppose that $K$ is a convex and $C$-embedded subspace of some product $E=\prod\left\{E_{\alpha}: \alpha \in A\right\}$ of separable Fréchet spaces. If $|A| \leq \omega$ then $K$ is a convex and closed subspace of a separable Fréchet space and hence is an absolute extensor (see, for example, [2]).

Now consider the case $|A|=\tau>\omega$. Without loss of generality we can suppose that each space $E_{\alpha}, \alpha \in A$, contains at least two points and consequently the space $E$ is homeomorphic to $R^{\tau}$ (we use Theorem A). By Proposition 1.14 from [3] there exists a functionally closed perfect 0 -invertible mapping $f: N^{A} \rightarrow E$ having a regular averaging operator (corresponding definitions that now for us have only technical sense can be found in [3]; we note only that $N$ denotes the discrete space of natural numbers). Since $\operatorname{dim} N^{\tau}=0$ and $K$ is a 
$C$-embedded $A E(0)$-subspace of $E$ there exists a mapping $h: N^{A} \rightarrow K$ such that $h\left|f^{-1}(K)=f\right| f^{-1}(K)$. Now we follow the proof of Theorem 3.2 from [3].

First let us introduce some notation: if $C \subseteq B \subseteq A$, then $\pi_{C}^{B}: \Pi\left\{E_{\alpha}: \alpha \in B\right\}$ $\rightarrow \Pi\left\{E_{\alpha}: \alpha \in C\right\}$ and $\pi_{B}: E \rightarrow \Pi\left\{E_{\alpha}: \alpha \in B\right\}$ are the natural projections. Put $K_{B}=\pi_{B}(K), K(B)=\pi_{B}^{-1}\left(K_{B}\right), p_{B}=\pi_{B} \mid K$, and $p_{C}^{B}=\pi_{C}^{B} \mid K_{B}$.

We say that a subset $B$ of $A$ is admissible (the last notion is equivalent to the notion of a weakly admissible subset from [3]) if $\pi_{B}(x)=p_{B} h f^{-1}(x)$ for each point $x \in K(B)$. As in [3] we can determine the following properties of admissible subsets:

(a) the union of arbitrary collection of admissible subsets is again an admissible subset;

(b) for any admissible subset $B$ the $K_{B}$ is closed and $C$-embedded in the product $\Pi\left\{E_{\alpha}: a \in B\right\}$;

(c) for any admissible subset $B$ the space $K_{B}$ is an $A E(0)$-space;

(d) for any admissible subsets $C$ and $B$ with $C \subseteq B$ the mappings $p_{B}$ and $p_{C}^{B}$ are 0 -soft;

(e) for any admissible subset $B$ the set $K_{B}$ is convex in the space $\Pi\left\{E_{\alpha}: a \in B\right\}$

(f) the family of all countable admissible subsets of $A$ is cofinal in the $\omega$-complete set $\exp _{\omega} A$.

Since $|A|=\tau$ we can write $A=\left\{a_{\alpha}: \alpha<\tau\right\}$. By (f) each point $a_{\alpha}$ of $A$ contained in some countable admissible subset $B_{\alpha}$. Put $A_{\alpha}=\bigcup\left\{B_{\beta}: \beta \leq \alpha\right\}$, $K_{\alpha}=K_{A_{\alpha}}, p_{\alpha}^{\alpha+1}=p_{A_{\alpha}}^{A_{\alpha+1}}(\alpha<\tau)$. It follows from the properties listed above for admissible subsets that the limit space of the naturally obtained well-ordered continuous spectrum $S=\left\{K_{\alpha}, p_{\alpha}^{\alpha+1}, \tau\right\}$ coincides with the given space $K$. Countability of the sets $B_{\alpha}$ implies, by Lemma 2, softness of the short projections of our spectrum. These facts are sufficient for us to conclude that $K$ is an $A E$-space. The lemma is proved.

Proof of Theorem B. We use a transfinite induction. For exactness we prove the following proposition: each infinite-dimensional locally convex $A E(0)$-space $E$ of topological weight $\tau \geq \omega$ is homeomorphic to $R^{\tau}$ (the additional condition of infinite-dimensionality is not restricting for us because each locally convex linear topological space of uncountable weight is infinite-dimensional). The starting point of induction (i.e., the case $\tau=\omega$ of the last proposition) is of course the Anderson-Kadets theorem. So we can suppose that our proposition is true for all locally convex $A E(0)$-spaces of weights $\lambda$ where $\omega \leq \lambda<\tau$ and consider a locally convex $A E(0)$-space $E$ of weight $\tau$. To prove that $E$ is homeomorphic to $R^{\tau}$ we can use a topological characterization of the last space. Let us recall some necessary definitions. Suppose that we have an $A E$ space $X$ of weight $\chi \geq \omega$. If $\chi=\omega$ then by the theorem of Torunczyk [11] the space $X$ is homeomorphic to $R^{\omega}$ if for an arbitrary mapping $g: Z \rightarrow X$ of any Polish space $Z$ in to $X$ and for any open covering $\mathscr{U}$ of $X$ there exists 
a closed embedding $h: Z \rightarrow X$ such that $h$ is $\mathscr{U}$-near to $g$. If $\chi>\omega$ then the characterization can be given as follows [3]: for an arbitrary mapping $g: Z \rightarrow$ $X$ of any space $Z$ with $R$-weight $\leq \chi$ (this means that $Z$ can be embedded into $R^{\chi}$ as a $C$-embedded subspace), for any cardinal number $\lambda$, with $\omega \leq \lambda<$ $\chi$, for any factorizing $\lambda$-spectrum $S_{X}^{\lambda}=\left\{X_{\alpha}, p_{\alpha}^{\beta}, A\right\}$ representing $X$, and for any index $\alpha \in A$ there exists a $C$-embedding $h: Z \rightarrow X$ such that $p_{\alpha} h=p_{\alpha} g$. It should be noted that in the last characterization we may consider only the class of spaces $Z R$-weights of which are strictly less than $\chi$ [3].

Thus consider an arbitrary space $Z$ the $R$-weight of which is equal to $\lambda$, where $\lambda<\tau$ (without loss of generality we can suppose that $\omega \leq \lambda$ ), any mapping $g: Z \rightarrow E$, any factorizing $\lambda$-spectrum $S_{E}=\left\{E_{\alpha}, p_{\alpha}^{\beta}, A\right\}$ with $\lim S_{E}=E$, and any index $\alpha \in A$. We want to construct a $C$-embedding $h: Z \rightarrow E$ such that $p_{\alpha} h=p_{\alpha} g$. Using Lemma 1 we can suppose without loss of generality that all spaces $E_{\alpha}$ are locally convex $A E(0)$-spaces of weight $\lambda$ and all limit projections $p_{\alpha}$ are linear 0-soft mappings. Moreover, by Lemma 3, $E$ is an $A E$-space. Consequently, by Theorem 1, we can suppose additionally that all spaces $E_{\alpha}$ are also $A E$-spaces and all limit projections $p_{\alpha}$ are linear and soft. Let us find an index $\beta \in A$ such that $\beta \geq \alpha$ and the projection $p_{\alpha}^{\beta}: E_{\beta} \rightarrow E_{\alpha}$ is a trivial bundle with fiber $R^{\lambda}$. Put $\alpha_{0}=\alpha$. Since the weight of $E_{\alpha_{0}}$ is strictly less than the weight of $E$ and the projection $p_{\alpha_{0}}: E \rightarrow E_{\alpha_{0}}$ is linear and open we can conclude that the fiber $\operatorname{ker} p_{\alpha_{0}}$ contains at least two points. Then we can choose an index $\alpha_{1}>\alpha_{0}$ such that $\operatorname{ker} p_{\alpha_{0}}^{\alpha_{1}}$ also contains at least two points. Suppose now that for every ordinal $\gamma$ with $\gamma<\delta$, where $\delta<\lambda$, we have already chosen indexes $\alpha_{\gamma} \in A$ in such a way that the following conditions are satisfied:

(a) if $\mu<\nu<\delta$, then $\alpha_{\mu}<\alpha_{\nu}$;

(b) if $\nu$ is a limit ordinal, then $\alpha_{\nu}=\sup \left\{\alpha_{\mu}: \mu<\nu\right\}$;

(c) the fiber $\operatorname{ker}_{\alpha_{\mu}}^{\alpha_{\nu}}$ contains at least two points $(\mu<\nu<\delta)$.

Now we construct an index $\alpha_{\delta}$ with the desired properties. If $\delta$ is a limit ordinal then we put $\alpha_{\delta}=\sup \left\{\alpha_{\gamma}: \gamma<\delta\right\}$. By the inequality $\delta<\lambda$ and $\lambda$ completeness of the spectrum $S$ we have that $\alpha_{\delta} \in A$. If $\delta=\gamma+1$ then index $\alpha_{\delta}$ can be constructed precisely in the same way as an index $\alpha_{1}$. Now consider $\beta=\sup \left\{\alpha_{\gamma}: \gamma<\lambda\right\}$ and note that $\beta \in A$. Consider the well-ordered spectrum $S^{\prime}=\left\{E_{\gamma}, p_{\alpha_{\gamma}}^{\alpha_{\gamma+1}}, \lambda\right\}$. Continuity of the spectrum $S$ implies that the limit space of the spectrum $S^{\prime}$ is naturally isomorphic to the space $E_{\beta}$ and the limit projection $\lim S^{\prime} \rightarrow E_{\alpha_{0}}=E_{\alpha}$ is isomorphic to the short projection $p_{\alpha}^{\beta}: E_{\beta} \rightarrow E_{\alpha}$ of the spectrum $S$. By the theorem of Bartle and Graves (see [2]; let us recall that the mapping $p_{\alpha}^{\beta}$ is linear and soft) this mapping is (topologically) a trivial bundle. At the same time it is easy to verify the fiber $\operatorname{ker} p_{\alpha}^{\beta}$ is homeomorphic to the product $\Pi\left\{\operatorname{ker} p_{\alpha_{\gamma}}^{\alpha_{\gamma+1}}: \gamma<\lambda\right\}$. Consequently, by conditions (a)-(c), the weight of $\operatorname{ker} p_{\alpha}^{\beta}$ is equal to $\lambda$. By the same conditions we can conclude 
that even if $\lambda=\omega$, the space is infinite-dimensional (in the other case we get a contradiction with the condition (c)). Consequently, by our hypothesis, the space $\operatorname{ker} p_{\alpha}^{\beta}$ is homeomorphic to $R^{\lambda}$. Moreover in this case the projection $p_{\alpha}^{\beta}$ can be topologically identified with the natural projection $\pi_{1}: E_{\alpha} \times R^{\lambda} \rightarrow E_{\alpha}$. Since the $R$-weight of the space $Z$ is equal to $\lambda$, there exists a $C$-embedding $i: Z \rightarrow R^{\lambda}$. Then it is easy to see the diagonal product $j$ of mappings $p_{\alpha} f$ and $i$ gives a $C$-embedding $j: Z \rightarrow E_{\alpha} \times R^{\lambda}=E_{\beta}$ for which the equality $p_{\alpha}^{\beta}=p_{\alpha} f$ holds. Let us fix an arbitrary lifting $k: E_{\beta} \rightarrow E$ of the soft limit projection $p_{\beta}$ (this means that $p_{\beta} k=\mathrm{id}_{E_{\beta}}$ ). Then the composition $h=k j$ is the desired $C$-embedding of $Z$ in $E$. Theorem B is proved.

The following proposition in the case of infinite-dimensional separable Fréchet spaces was proved by Klee and Corson (see [2]).

Corollary 2. Every closed convex body in a locally convex $A E(0)$-space of weight $\tau>\omega$ is homeomorphic to $R^{\tau}$.

Proof. Let $X$ be a closed and convex body in a locally convex $A E(0)$-space $E$ of weight $\tau>\omega$. Since $\operatorname{int}(X) \neq \varnothing$ we can conclude that $X=\operatorname{cl}(\operatorname{int}(X))$. By Theorem B the space $E$ is homeomorphic to $R^{\tau}$ and consequently $X$ is a functionally closed (this means that there exists some function $f \in C(E)$ such that $f^{-1}(0)=X$ ) and $C$-embedded subspace of $E$. By Corollary 4.2 from [3] $X$ is an $A E(0)$-space. Lemma 3 implies that in this case $X$ is even an $A E$-space. Hence by the corresponding result from [4] $X$ is homeomorphic to $R^{\tau}$. The proof is complete.

The following proposition in the case of metrizable spaces closely relates with the theorem of Mazur-Strenbach [2].

Corollary 3. Every linear $G_{\delta}$-subspace of a locally convex $A E(0)$-space of weight $\tau>\omega$ is homeomorphic to $R^{\tau}$.

Proof. Let $X$ be a linear $G_{\delta}$-subspace of locally convex $A E(0)$-space $E$ of weight $\tau>\omega$. Put $Y=\operatorname{cl}(X)$. As in the proof of Corollary 2 we can conclude that $Y$ is homeomorphic to $R^{\tau}$. Consequently it is sufficient to show that $X=Y$. In fact, if there exists a point $y \in Y \backslash X$ then the translate $X+y$ would be a dense $G_{\delta}$-subspace of $Y$ and $(X+y) \cap X=\varnothing$, which is contradictory with the Baire property in $Y \cong R^{\tau}$.

The following proposition can be proved in a completely similar way as Theorem B (and consequently its proof is omitted here).

Proposition 1. The image of a continuous linear retraction defined on the product of an arbitrary family of separable Fréchet spaces is isomorphic to the product of some family of separable Fréchet spaces.

Finally it should be observed that using the topological characterization of trivial bundle with fiber $R^{\tau}$ (see [5]; in the case $\tau=\omega$ this characterization 
was obtained by Torunczyk-unpublished result cited in [5]) it is possible to prove that each linear 0 -soft mapping $f$ between locally convex $A E(0)$-spaces with $w(\operatorname{ker} f)=\tau \geq \omega$ is topologically a trivial bundle with fiber $R^{\tau}$ (note that in Lemma 2 only mappings having a Polish kernel were considered).

\section{REFERENCES}

1. P. Alexandrov and P. Urysohn, Uber nulldimensional Punktmengen, Math. Ann. 98 (1927/ 1928), 89-106.

2. C. Bessaga and A. Pelczynski, Selected topics in infinite-dimensional topology, PWN, Warsaw, 1975.

3. A. Chigogidze, Noncompact absolute extensors in dimension $n, n$-soft mappings and their applications, Izvestia Acad. Nauk USSR 50 (1986), 156-180.

4. __ On the structure of nonmetrizable AE(0)-spaces, Math. Z. 41 (1987), 406-411.

5. __ Trivial bundles and near homeomorphisms, Fund. Math. 132 (1989), 89-98.

6. A. N. Dranishnikov, Absolute extensors in dimension $n$ and dimension-raising $n$-soft mappings, Uspekhi Mat. Nauk 39 (1984), 55-95.

7. R. Engelking, General topology, PWN, Warsaw, 1977.

8. V. V. Fedorchuk, On open mappings, Uspekhi Mat. Nauk 37 (1982), 187-188.

9. R. Haydon, On a problem of Pelczynski: Milutin spaces, Dugundji spaces and $A E(0-\operatorname{dim})$, Studia Math. 52 (1974), 23-31.

10. E. V. Shchepin, Functors and uncountable powers of compacta, Uspekhi Mat. Nauk 36 (1981), 3-62.

11. H. Torunczyk, Characterizing Hilbert space topology, Fund. Math. 111 (1981), 247-262.

Mathematics Department, Chair of General Topology and Topology, Moscow State UNIVERSITY, MOSCOW 119899, USSR 